\title{
DÜBLIN
}

Technological University Dublin

ARROW@TU Dublin

2014-8

\section{A Soft Condensed Matter Approach Towards Mathematical Modelling of Mass Transport and Swelling in Food Grains}

\author{
Michael Chapwanya \\ University of Pretoria
}

N. Misra

Technological University Dublin, misra.nrusimhanath@tudublin.ie

Follow this and additional works at: https://arrow.tudublin.ie/schfsehart

Part of the Complex Fluids Commons, Condensed Matter Physics Commons, Food Processing Commons, Partial Differential Equations Commons, Polymer and Organic Materials Commons, Polymer Science Commons, Thermodynamics Commons, and the Transport Phenomena Commons

\section{Recommended Citation}

M. Chapwanya, N.N. Misra (2015) A soft condensed matter approach towards mathematical modelling of mass transport and swelling in food grains, Journal of Food Engineering, 145:37-44. http://dx.doi.org/ 10.1016/j.jfoodeng.2014.08.010

This Article is brought to you for free and open access by the School of Food Science and Environmental Health at ARROW@TU Dublin. It has been accepted for inclusion in Articles by an authorized administrator of ARROW@TU Dublin. For more information, please contact arrow.admin@tudublin.ie, aisling.coyne@tudublin.ie, gerard.connolly@tudublin.ie.

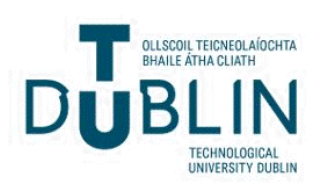


1 A soft condensed matter approach towards mathematical modelling of mass transport and swelling in food grains

$4{ }^{a}$ Department of Mathematics 83 Applied Mathematics, University of Pretoria, Pretoria 0002, South Africa

$5 \quad{ }^{b}$ School of Food Science \& Environmental Health, Dublin Institute of Technology, Dublin 1, Ireland.

\section{Abstract}

Soft condensed matter (SCM) physics has recently gained importance for a large class of engineering materials. The treatment of food materials from a soft matter perspective, however, is only at the surface and is gaining importance for understanding the complex phenomena and structure of foods. In this work, we present a theoretical treatment of navy beans from a SCM perspective to describe the hydration kinetics. We solve the transport equations within a porous matrix and employ the Flory-Huggin's equation for polymersolvent mixture to balance the osmotic pressure. The swelling of the legume seed is modelled as a moving boundary with an explicit transient equation. The model exhibits a good agreement with the experimental observations and is capable of explaining the stages of hydration. Sensitivity analysis indicated that the degree of hydration is dependent on the bean size and is also sensitive to the selection of the intrinsic permeability of the bean.

7 Keywords: Soft Condensed Matter, Hydration, Swelling, Flory-Huggins, Mass Transfer, 8 Navy Bean

\section{1. Introduction}

Soaking and hydration of legumes and cereals is an important unit operation in the grain processing industries. For example, legumes such as navy bean and kidney bean are often hydrated prior to canning operations. Hydration of beans decreases the cooking time,

\footnotetext{
${ }^{*}$ Corresponding author

Email address: misra.nrusimhanath@dit.ie (N.N. Misra)
} 
minimizes losses and improves the nutritional quality and protein digestibility of the cooked product (Wang et al, 1979; Abd El-Hady and Habiba, 2003). The problem of mass transfer during hydration of food grains has been treated both experimentally and theoretically, and in studies combining both approaches (Zanella-Díaz et al, 2014; Cozzolino et al, 2013; Ghafoor et al, 2014; Nicolin et al, 2012; Bello et al, 2010; Mohoric et al, 2004; Peleg, 1988; Hsu, 1983). Most of the mathematical descriptions reported in literature are either data driven regression models describing hydration kinetics or are based on simple fickian diffusion. Diffusion in many legumes (and cereals) cannot be described adequately by a simple concentration dependent form of Fick's diffusion equation, especially when these undergo swelling (or large deformation in geometry). Such conventional approaches fail to capture the finer details of food structure and their dynamics. Because of the complexity of food systems, interdisciplinary scientific approaches are needed to enable demanding developments (Ubbink and Mezzenga, 2006; Ubbink et al, 2008).

Soft matter physics focusing on description of an increasingly important class of materials that encompasses polymers, liquid crystals, complex fluids, organic-inorganic hybrids, foams, gels and the whole area of colloidal science is a contemporary area of research with several opportunities. Soft matter science plays an important role in a wide variety of processes and applications, examples of which include polymer swelling, phase separation, transport and delivery of drugs etc. The principles of soft matter physics are equally applicable to many food systems. Mezzenga et al (2005) reviewed the nature of foods from a perspective of soft condensed matter physics. The details of structural changes at various scales in food systems often needs experimental and/or theoretical tools of soft matter physics, which are not fully adapted to food systems (Mezzenga, 2007; Mezzenga et al, 2005). An exposition of the potential of soft matter physics for explaining the complex food processes and structuring at various scales is also provided in van der Sman and van der Goot (2009) and van der Sman (2012).

About 60 years ago, Flory and Huggins independently proposed the lattice model to treat the mixing enthalpy and entropy of polymers in a very straightforward way (Huggins, 1942a,b; Flory, 1953). Although many other models have been developed to describe the 
thermodynamics of polymer systems, the original Flory-Huggins lattice theory always give a very clear and straightforward physical picture (Han and Akcasu, 2011). However, it is worthwhile noting that the Flory-Huggins theory also is based on some assumptions that are often not valid. This includes the assumption that volume changes are not incurred upon mixing the polymer and solvent. It is also supposed that the polymer chain can be modelled on a lattice, which excludes contributions to the entropy from chain flexibility, and specific solvent-polymer interactions are ruled-out (Hamley, 2007). In an early work, van der Sman (2007) deduced an excellent modelling framework based on soft condensed matter perspective to explain the heat and mass transport during cooking of meat. This was based on the Flory-Rehner theory for pressure driven mass-transport in swelling or shrinking gels.

The model that we present in this paper is an attempt at advancing the basis of the theoretical modeling of mass transport during hydration of porous foods with a soft matter perspective. The model described herein shares an integrated approach between the statistical thermodynamics based Flory-Huggins theory and the continuum mechanics of fluid transport in porous media. We take an example case of hydration of navy bean, which is modelled as a saturated porous media undergoing large deformation by swelling. We are including a comparison of the simulation results with experimental data as model validation and an illustration of the model application, while also reporting on the sensitivity of the model to selected parameters. By means of the latter, we attempt to both analyse the influence of the natural variability in food properties on model predictions as well as gauge the sensitivity of the model to potential errors in parameter estimation.

\section{Problem description}

Figure 1, provides a summary of the geometric domain of the bean under consideration. The geometry of the navy bean can be described as a porous scalene ellipsoid, which is capable of undergoing global deformation. For the present study, we also assume that the pores are ideally filled with water which simplifies the problem to a saturated porous media case. We assume that the complex structural elements of navy bean, mainly proteins and 
carbohydrates can be approximated as ideal polymers. When applying principles of soft condensed matter physics to foods, it may be noted that polysaccharides and proteins are to foods what polymers are to soft condensed matter (Mezzenga et al, 2005). Notably, navy

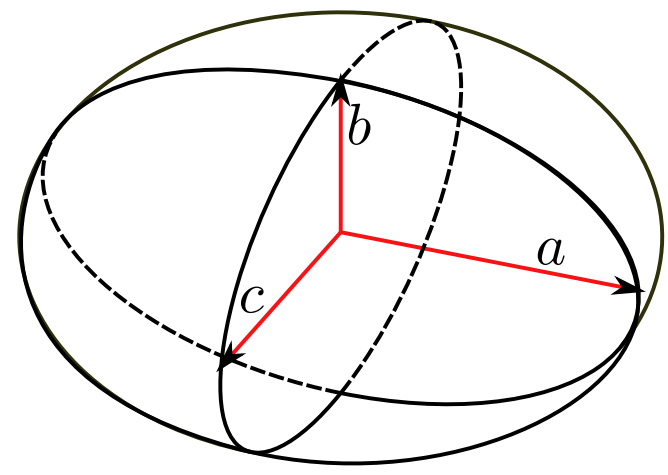

(a) Ellipsoid geometry of navy bean.

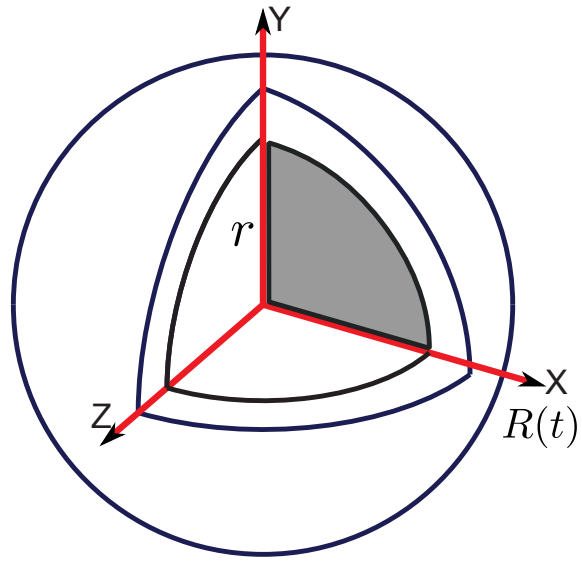

(b) Equivalent geometry for the model.

Figure 1: Schematic illustration of (a) the ellipsoid geometry of a navy bean grain and (b) the equivalent sphere concept with model boundaries.

We now simplify the problem geometry, by assuming that bean is spherical in shape and

$$
r=\left(\frac{G_{m}+S_{m}+A_{m}}{6}\right)
$$


Herein, $G_{m}=2(a b c)^{\frac{1}{3}}, A_{m}=\left(\frac{2 a+2 b+2 c}{3}\right)$, and $S_{m}=\left(\frac{4 a b+4 b c+4 c a}{3}\right)^{\frac{1}{3}}$ are the geometric mean diameter, arithmetic mean diamteter, and square mean diameter, respectively (Mohsenin, 1986). In accordance with Ghafoor et al (2014), we take the temperature to be constant $\left(16{ }^{\circ} \mathrm{C}\right)$ throughout the duration of soaking and therefore, temperatures capable of causing gelatinisation are not encountered. Finally, we define the volume fraction of the solids in the bean to be $\phi$, and to satisfy the criteria of saturation, we have $(1-\phi)$ as the volume fraction of the liquid water.

\section{Mathematical model}

In this section a mathematical model based on the Flory-Huggins theory for hydration of Navy bean is presented. Part of the modeling approach has been presented elsewhere in the literature in the context of biofilms (Winstanley et al, 2011). For completeness, we summarize the equations here. Mass conservation of the polymer is given by

$$
\left(\rho_{s} \phi\right)_{t}+\nabla \cdot\left[\rho_{s} \phi \mathbf{v}\right]=0
$$

where $\rho_{s}\left[\mathrm{Kg} \mathrm{m}^{-3}\right]$ is the averaged phase density and $\mathbf{v}\left[\mathrm{m} \mathrm{s}^{-1}\right]$ is the protein velocity. Similarly, conservation of the liquid is given by

$$
(\rho[1-\phi])_{t}+\nabla \cdot[\rho(1-\phi) \mathbf{w}]=0
$$

98 where $\rho\left[\mathrm{Kg} \mathrm{m}^{-3}\right]$ is the averaged water density and $\mathbf{w}\left[\mathrm{m} \mathrm{s}^{-1}\right]$ is the water velocity.

The bean is assumed to be a porous structure, hence the momentum equations as given by Darcy's law holds. We relate the liquid velocity to the liquid pressure, $p[\mathrm{~Pa}]$, via

$$
-(1-\phi) \nabla p+\frac{\mu(1-\phi)^{2}}{k}(\mathbf{v}-\mathbf{w})=0
$$

01 where $\mu$ is the water viscosity, $k$ is the grain permeability. By analogy with (4), the momentum equation for the polymer takes the form

$$
-\phi \nabla p_{s}-\frac{\mu(1-\phi)^{2}}{k}(\mathbf{v}-\mathbf{w})=0
$$


where $p_{s}[\mathrm{~Pa}]$, is the pressure of the polymer. Note that our approach in dropping the viscous stress for equations (8) and (9) is consistent with the the work of Winstanley et al (2011), where the viscous stress was observed to be negligible compared to osmotic stress and pressure. The interaction pressure, which is the pressure difference between the polymer pressure and the water pressure, is given by

$$
p_{s}-p=\psi(\phi)
$$

Note that, a polymer in contact with water will swell (or shrink) to equilibrate the total osmotic pressure - and swelling (in the current context) will give rise to changes in $\phi$. It has been recognised that the balance equations can be generalized to have a proper coupling to thermodynamics, so that they have the correct driving forces for the transport phenomena (van der Sman, 2012). Keeping this in mind, as will be shown next, here we adopt the Flory-Huggins theory to relate the osmotic pressure to the polymer volume fraction, i.e., $\psi=\psi(\phi)$. At this point it may be noted that the processes of diffusion and diffusivity are fundamentally different in nature, although they are formally described by the same type of mathematics.

We now manifest the Flory-Huggins free energy per unit volume, as the osmotic pressure term (see c.f. Hill (1987)), which is the additional pressure that is required to equilibrate the solid (polymer) volume fraction with pure water. This is given by-

$$
\psi=-\frac{R T}{V}\left[\ln (1-\phi)+\left(1-\frac{1}{n}\right) \phi+\chi \phi^{2}\right]
$$

where $n$ is the ratio of molar volumes of solute (protein) and solvent (water), $\chi$ is the interaction parameter between the polymer and the solvent (water), $R\left[\mathrm{~J} \mathrm{~mol}^{-1} \mathrm{~K}^{-1}\right]$ is the gas constant, $T[\mathrm{~K}]$ is the temperature and $V\left[\mathrm{~m}^{3} \mathrm{~mol}^{-1}\right]$ is the molar volume of water. The underpinning principles of the theory are backed up by strong thermodynamic basis and the lattice theory, a good discussion of which can be found in Hill (1987). From a thermodynamic viewpoint, the parameter $\chi$ is a measure of the interaction enthalpy per solvent (water) molecule. Proceeding further, if $n$ is sufficiently large $(n \rightarrow \infty)$, which is a valid assumption for the case of most foods, including navy bean, equation (6) can be 
128

\subsection{Model summary}

Assuming incompressibility, we summarize the equations as follows

$$
\begin{aligned}
-f \phi(1-\phi)(\mathbf{v}-\mathbf{w})-\phi \nabla \psi-\phi \nabla p & =0, \\
f \phi(1-\phi)(\mathbf{v}-\mathbf{w})-(1-\phi) \nabla p & =0, \\
\phi_{t}+\nabla \cdot[\phi \mathbf{v}] & =0, \\
-\phi_{t}+\nabla \cdot[(1-\phi) \mathbf{w}] & =0, \\
\psi & =-\frac{R T}{V}\left[\ln (1-\phi)+\phi+\chi \phi^{2}\right],
\end{aligned}
$$

where $f=\frac{\mu(1-\phi)}{k \phi}\left[\mathrm{Pas} \mathrm{m}^{-2}\right]$ is the interfacial drag term. The model above incorporates the momentum balance (Darcy's law) for each phase (equations (8) and (9)), the osmotic pressure equation (Flory-Huggins equation (12)) relating the chemical potential of the solvent (water) the solute (polymer), and mass conservation for each phase (equations (10) and (11)).

The swelling/shrinking pressure is proportional to $-\nabla \psi=-\psi^{\prime}(\phi) \nabla \phi$ where

$$
\psi^{\prime}(\phi)=\frac{R T}{V} \frac{2 \phi^{2}}{1-\phi}\left[\left(\chi-\frac{1}{2}\right)-\chi \phi\right] .
$$

Here we consider $\psi^{\prime}>0$ where a polymer in contact with a solvent will swell. We note that for $\phi \in[0,1]$, there is a minimum at $\phi^{*}=\left(\chi-\frac{1}{2}\right) / \chi$ with $\chi>\frac{1}{2}$, as can be observed in Fig. 2. The above relation can therefore be used for tracking the equilibrium moisture interface over a moving boundary.

After some algebraic simplification, the system of equations (8) - (12) can be reduced to

$$
\begin{aligned}
\phi_{t} & =\nabla \cdot[(1-\phi) \mathbf{w}], \\
\mathbf{w} & =\frac{\phi}{f(\phi)} \psi^{\prime}(\phi) \nabla \phi, \\
\psi & =-\frac{R T}{V}\left[\ln (1-\phi)+\phi+\chi \phi^{2}\right] .
\end{aligned}
$$




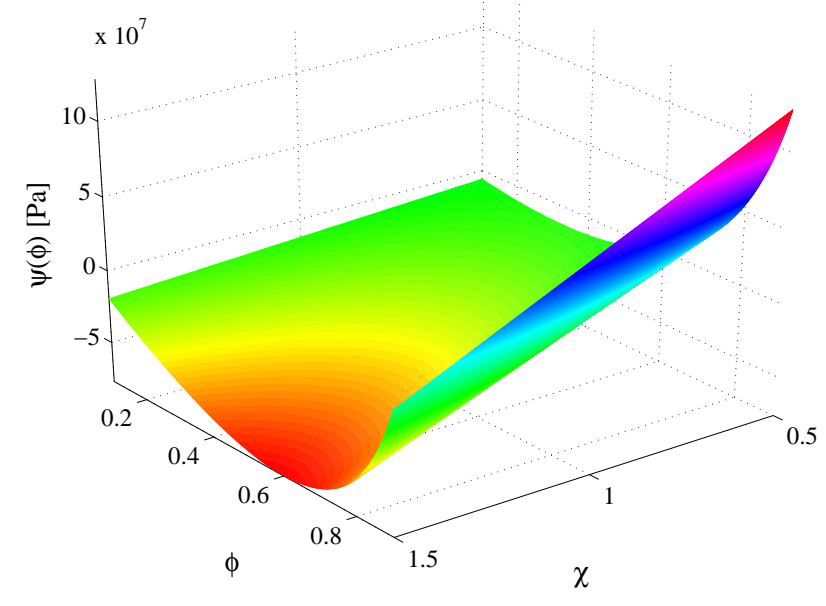

Figure 2: An illustration of the relationship between osmotic pressure and polymer fraction.

Assuming one dimensional spherical coordinate system, then $\phi(r, t)$ is shown to be governed by the Fick's second Law of diffusion

$$
\frac{\partial \phi}{\partial t}=\frac{1}{r^{2}} \frac{\partial}{\partial r}\left[r^{2} D(\phi) \frac{\partial \phi}{\partial r}\right],
$$

where the moisture diffusivity term is given by

$$
D(\phi)=D_{0} \frac{2 \phi^{3}}{1-\phi}\left[\chi \phi-\left(\chi-\frac{1}{2}\right)\right]
$$

and $D_{0}=\frac{R T k}{\mu V}$. We now require that $\chi \phi-\left(\chi-\frac{1}{2}\right)>0$ or $\phi^{*}<\phi<\phi_{e}$. The Flory's interaction parameter, $\chi$ in this range corresponds to approx. [0.5-1.5], which also complies with the values reported in Jin et al (2014). This will be used later to motivate the choice of boundary condition at the interface between free water and the porous structure. This constrain also arises from the peculiar nature of $\chi$ as the parameter dictating the phase separations in polymer-solvent systems, a comment on which is appropriate. The critical value of $\chi$ (denoted as $\chi_{c}$ ) for miscibility of a polymer in a solvent is approximately 0.5. For values of $\chi$ less than 0.5 the polymer will be soluble in the solvent and loss of solids (leaching) will occur. However, the analogous polymer-solvent theory for navy bean assuming no loss of solids (solute) requires that the biopolymer matrix be insoluble in the water, i.e. a poor solvent behaviour. Therefore, our assumption of $\chi>\frac{1}{2}$ is physically valid, for only with this constrain the polymer will not be soluble in the solvent (Flory, 1953; Hill, 1987). 
It is worth noting the similarities between the derived model and the literature; see for example Bello et al (2010); Davey et al (2002); Weinstein and Bennethum (2006); Nicolin et al (2012). In particular, Davey et al (2002) derived a nonlinear diffusion equation governing the swelling of a cereal grain. However, the model here deviates from Davey et al (2002) in the choice of the diffusivity function. While their choice is motivated by an empirical exponential function, here the function comes naturally from the Flory-Huggins theory. A model based on thermodynamics arguments and Flory-Huggins theory was also presented by Weinstein and Bennethum (2006). The resulting nonlinear diffusion equation can be reduced to the model derived here with the condition that $\chi=0$ and an appropriate choice of the permeability function. However, as we have observed earlier, the limit $\chi=0$ does not make any physical sense.

From a mathematical point of view, the presented model is a moving boundary problem and requires an extra condition for the location of the boundary $r=R(t)$. As the water is absorbed, the grain swells and there is a change of mass inside the grain. From here forthwith, we define $\phi=1-\theta$, so that $\theta$ defines the volume fraction of water and $1-\theta$ the volume fraction of the polymer. Using the new variable we have

$$
\frac{\partial \theta}{\partial t}=\frac{1}{r^{2}} \frac{\partial}{\partial r}\left[r^{2} D(\theta) \frac{\partial \theta}{\partial r}\right]
$$

$$
D(\theta)=D_{0} \frac{2(1-\theta)^{3}}{\theta}\left[\chi(1-\theta)-\left(\chi-\frac{1}{2}\right)\right],
$$

with $\theta>0$. Integrating (18) on $r \in[0, R(t)]$ and on application of Leibniz rule, we have

$$
R^{2} \frac{d R}{d t}=\left.\frac{D\left(\theta_{e}\right) R^{2}}{1-\theta_{e}} \frac{\partial \theta}{\partial r}\right|_{r=R(t)}+\frac{1}{1-\theta_{e}} \frac{d}{d t}\left[\int_{0}^{R(t)}(1-\theta) r^{2} d r\right],
$$

73 where we have used symmetry and the fact that $\theta_{r}=0$ on $r=0$. The middle term gives 174 the flux of water through the grain and the last term gives the dissolution rate. Here there is no loss of solid during hydration, hence we set

$$
\frac{d}{d t}\left[\int_{0}^{R(t)}(1-\theta) r^{2} d r\right]=0
$$


so that the moving boundary is described by

$$
\frac{d R}{d t}=\left.\frac{D\left(\theta_{e}\right)}{1-\theta_{e}} \frac{\partial \theta}{\partial r}\right|_{r=R(t)} .
$$

\subsection{Boundary Conditions}

As initial condition, we know that $\theta(r, 0)=\theta_{0}$ under the assumption that the moisture distribution inside the bean is isotropic. We now specify the boundary conditions as follows

$$
\begin{aligned}
& \frac{\partial \theta}{\partial r}=0, \quad \text { on } \quad r=0, \\
& \theta=\theta_{e}, \quad \text { on } \quad r=R(t) .
\end{aligned}
$$

The volume averaged moisture content at each time step, $t[\mathrm{~s}]$ is calculated from equation (25), as given by Ruiz et al (2008),

$$
M(t)=\frac{3}{R(t)^{3}} \int_{0}^{R(t)} R(t)^{2} \theta(R, t) d r,
$$

where $R(t)$ is the radius at time $t[\mathrm{~s}]$. For validation of the model, we employ the experimental results from a previous work Ghafoor et al (2014) and set all parameters as per Table 1, unless otherwise explicitly stated. The moisture content on dry basis given in Ghafoor et al (2014) was transformed into volume fraction of water, $\theta(r, t)$, using the following relation

$$
M=\frac{\rho \theta}{\rho_{s}(1-\theta)},
$$

\section{Nondimensionalisation}

We introduce the following scales

$$
r \sim \ell, \quad s \sim \ell, \quad R \sim \ell, \quad D \sim D_{0}, \quad t \sim \frac{\ell^{2}}{D_{0}},
$$

where $\ell$ is the initial bean radius. The advantage of this process is that we now have to solve the model on $[0, R(t)]$ with $R(t) \geq 1$. In dimensionless form, we consider

$$
\begin{aligned}
\frac{\partial \theta}{\partial t} & =\frac{1}{r^{2}} \frac{\partial}{\partial r}\left[r^{2} D(\theta) \frac{\partial \theta}{\partial r}\right], \quad \forall(r, t) \in(0, R(t)) \times(0, \infty), \\
\theta(r, 0) & =\theta_{0},
\end{aligned}
$$


Table 1: Typical values of the parameters employed for the simulations and model validation.

\begin{tabular}{|c|c|c|c|c|}
\hline Symbol & Definition & Value & & Source \\
\hline$\theta_{0}$ & Initial water fraction & 0.12 & & Ghafoor et al (2014) \\
\hline$\theta_{e}$ & Equilibrium water fraction & 0.60 & & Ghafoor et al (2014) \\
\hline$T$ & Temperature & 289.15 & $\mathrm{~K}$ & Ghafoor et al (2014) \\
\hline$\ell$ & Navy bean initial radius & 0.003 & $\mathrm{~m}$ & Ghafoor et al (2014) \\
\hline$\rho_{s}$ & Polymer matrix density & 1376 & $\mathrm{~kg} / \mathrm{m}^{3}$ & Bellido et al (2003) \\
\hline$\rho$ & Density of water & 999.1 & $\mathrm{~kg} / \mathrm{m}^{3}$ & \\
\hline$\mu$ & Viscosity of water & $1.109 \times 10^{-3}$ & $\mathrm{~Pa} \mathrm{~s}$ & \\
\hline$k$ & Permeability & $3.1 \times 10^{-21}$ (approx.) & $\mathrm{m}^{2}$ & Warning et al (2014) \\
\hline$\chi$ & Interaction parameter & 0.51 & & Jin et al (2014) \\
\hline$R$ & Gas constant & 8.314 & $\mathrm{~J} / \mathrm{mol} / \mathrm{K}$ & \\
\hline$V$ & Molar volume of water & $18.02 \times 10^{-6}$ & $\mathrm{~m}^{3} / \mathrm{mol}$ & \\
\hline$D_{0}$ & $R T k / \mu V$ & $4.81 \times 10^{-10}$ & $\mathrm{~m}^{2} / \mathrm{s}$ & \\
\hline
\end{tabular}

subject to

$$
\begin{aligned}
\frac{\partial \theta}{\partial r}(0, t) & =0, \\
\theta(R, t) & =\theta_{e}, \quad \text { and } \quad \frac{d R}{d t}=\left.\frac{D\left(\theta_{e}\right)}{1-\theta_{e}} \frac{\partial \theta}{\partial r}\right|_{r=R(t)},
\end{aligned}
$$

187 where

$$
D(\theta)=\frac{2(1-\theta)^{3}}{\theta}\left[\chi(1-\theta)-\left(\chi-\frac{1}{2}\right)\right]
$$

188 While some mathematical insight can be obtained from an equivalent two moving boundary 189 problem as in Davey et al (2002), here no analytical approximation is possible. Hence we 190 proceed to solve the problem numerically. 


\section{Numerical simulations and model validation}

The model is discretized in space using finite volume approach on the domain $[0, R(t)]$ with $N$ uniform grid cells of width $\Delta r=R(t) / N$ with cell centers $r_{i \pm \frac{1}{2}}=r_{i} \pm \frac{\Delta r}{2}$ for $i=1,2, \cdots, N$. Using the notation consistent with the finite volume literature, we have the scheme

$$
\frac{\partial \theta_{i}}{\partial t}=\frac{1}{r_{i}^{2} \Delta r}\left[r_{i+1 / 2}^{2} D_{i+1 / 2} \frac{\theta_{i+1}-\theta_{i}}{\Delta r}-r_{i-1 / 2}^{2} D_{i-1 / 2} \frac{\theta_{i}-\theta_{i-1}}{\Delta r}\right] .
$$

for equation (28), and

$$
\frac{d R}{d t}=\left.\frac{D\left(\theta_{e}\right)}{1-\theta_{e}} \frac{\theta_{i+1 / 2}-\theta_{i-1 / 2}}{\Delta r}\right|_{r_{i}=R(t)}
$$

for the moving boundary (31). The same approach is used for the boundary conditions and the resulting scheme is second order in space. The solution code scripted in MATLAB (The MathWorks, MA, USA) was run on a $3.0 \mathrm{GHz}$ Intel Core i7 processor. The system of nonlinear ODEs are integrated with Matlab's standard stiff solver ODE15s, with a relative tolerance values of $1 \times 10^{-12}$. The resolution algorithm of ODE15s is based on the numerical differentiation formula method (improved version of the implicit Backward Differentiation Formula (BDF) method). For all the simulations presented in this paper, we used $N=201$ and we found no influence on the results upon rerunning the simulations with increased number of nodes.

For evaluating the accuracy of the models, we employ the statistical criterion of the Root Mean Squared Error (RMSE) given by equation (35)

$$
R M S E=\sqrt{\frac{\sum_{i=1}^{n_{t}}\left(\theta_{e}-\theta_{p}\right)^{2}}{n_{t}}}
$$

where $\theta_{e}$ denotes the experimental observations, $\theta_{p}$ the predicted values and $n_{t}$ the total number of data points.

\subsection{Base case simulations}

We now compare our results with the experimental data reported by Ghafoor et al (2014), who studied the hydration of navy beans in water at $16{ }^{\circ} \mathrm{C}$. Figure 3 (a) provides a comparison of the model predictions and the experimental data. The model clearly provides a good 
prediction of the hydration kinetics, within a reasonably small error as observed from the RMSE value of 0.081. At the beginning of the water absorption process, the relative mass of water absorbed by the bean cotyledon strongly increased with the hydration time and then saturated. The exponential behaviour indicates that the water absorption is caused by the gradient of the matrix potential between the dry cotyledon structure and the water environment (Golonka et al, 2002). The volume fraction of the liquid representing the moisture content tends to approximately a value of 0.6 after $15 \mathrm{~h}$ of soaking. In addition, we note that the radius of the representative bean follows a similar trend and swells to a maximum radii of $\sim 3.9 \mathrm{~mm}$, which is in excellent agreement with the experimental value of $3.92 \mathrm{~mm}$. We wish to highlight that unlike, the work of Bakalis et al (2009) and Hsu (1983) who employ experimental observations and empirical equations respectively for the bean swelling, our approach accurately predicts the radii as a function of the instantaneous moisture. The deviation from the experimental values during the initial phase of hydration can be ascribed to our ignorance of resistance offered by the seed coat. This fact is experimentally demonstrated elsewhere in the context of soybean hydration (Meyer et al, 2006).

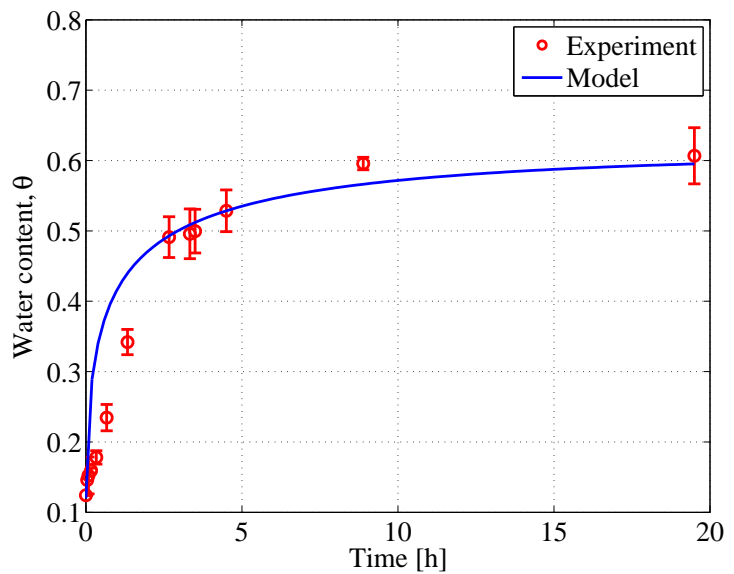

(a) Water content.

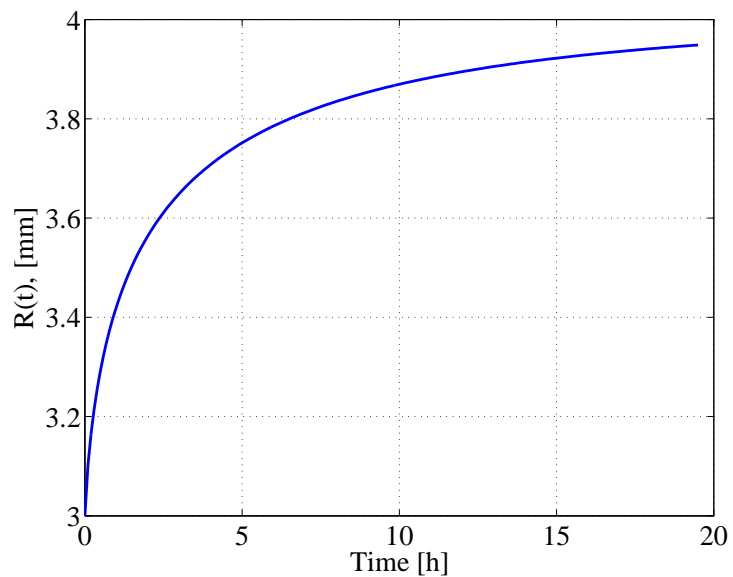

(b) Moving boundary.

Figure 3: Profiles for water content and the radius of the bean with time. 


\subsection{Degree of hydration}

An earlier work on cooking of grains focused on the degree of gelatinisation of a rice kernel and the significant feature of this model was the presence of two moving boundaries. One describing the change in grain size and the second boundary described the gelatinisation front (Davey et al, 2002). Mathematically, these represent a class of Stefan's problem where no phase change is involved (see, for example Barry and Caunce (2008)). Since cooking temperatures leading to gelatinisation are never encountered for simple hydration, we are left with one moving boundary for the radius and a transient species diffusion. Equivalently, here we implicitly locate the degree of hydration by tracking the front where the moisture content is $\theta_{w}$. This approach is more physically representative since we are effectively allowing moisture variations beyond the wetting front. We present these relations via. a contour plot in Figure 4. The rapid hydration in the initial phase can be ascribed to the creation of free volumes inside the grains, while the slow water uptake in later phases is an outcome of the rearrangement of structural elements. Direct experimental observations using Positron Annihilation Spectroscopy (PAS) and proton Nuclear Magnetic Relaxation (NMR) reported earlier also support our hypothesis and explanation (Golonka et al, 2002). Golonka et al (2002) reported that the hydration of the cotyledon structure evokes reduction of the surface and interior tension, and loosens structure of the bean samples. When the hydration reaches the slower phase, a rearrangement of the cotyledon microstructure occurs and the number and dimensions of the free volume regions does not change significantly.

\subsection{Sensitivity to bean dimensions}

Experimental measurement of the initial bean dimensions of several beans and the average values $a, b$ and $c$ were in the range $8.9 \pm 0.39 \mathrm{~mm}, 5.04 \pm 0.15 \mathrm{~mm}$ and $5.9 \pm 0.28 \mathrm{~mm}$ respectively, see Ghafoor et al (2014). The bean dimensions after a 19 h soaking duration were found to be in the range $12.94 \pm 0.47 \mathrm{~mm}, 6.32 \pm 0.31 \mathrm{~mm}$ and $7.54 \pm 0.21 \mathrm{~mm}$ respectively. These values corresponded to an initial and final radii of the equivalent sphere in the range $3 \mathrm{~mm}$ and $4 \mathrm{~mm}$ respectively. Differences in bean dimensions affect the degree of hydration, especially for shorter hydration times, which nevertheless, is overcome with 


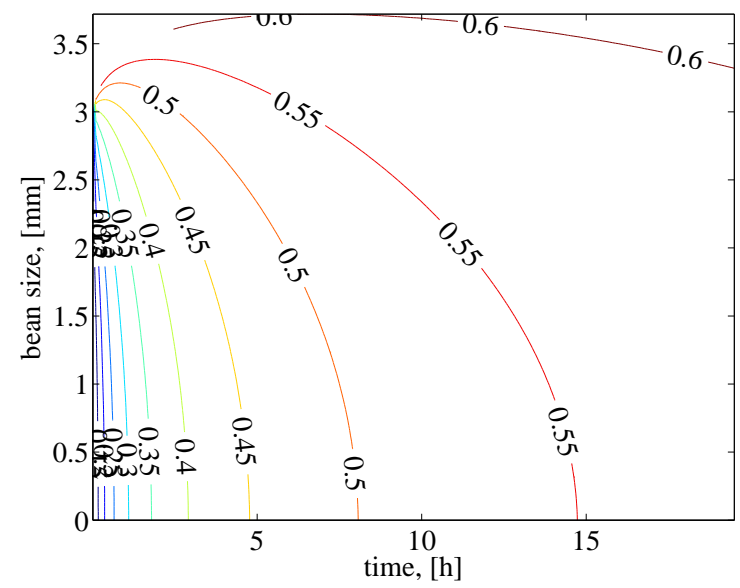

Figure 4: The isolines represent the degree of hydration of the grain at a given time.

standard overnight soaking practice. We performed sensitivity analysis for the degree of hydration to bean dimensions in the range 2.5 to $4.0 \mathrm{~mm}$. The results are summarised in Figure 5. Firstly, we note that beans with higher initial radii swell to higher degree and this was also observed during the experiments (Ghafoor et al, 2014). Next, the moisture equilibration takes more time for larger beans. However, longer soaking times allow to reach near equilibrium moisture distribution.

\subsection{Sensitivity to medium permeability}

When considering the moisture diffusion in a single bean, the porosity, pore network size and distribution largely define the rate of hydration. Since we chose porosity as a macroscopic variable in our model, the above factors are ruled by the intrinsic permeability in this framework. Datta (2006) highlighted that when physics based mechanistic models involving pressure driven flow are employed with a clear definition of the individual modes of transport processes, the selection of permeability data becomes important. Considering that we did not find experimentally measured permeability data for navy beans, we approximated the intrinsic permeability from that of parboiled rice grains and those reported for other inert materials (Warning et al, 2014; Datta, 2006). The permeability for heat treated parboiled rice grain was recently reported to be in the order of $10^{-20} \mathrm{~m}^{2}$ using Lattice-Boltzmann simulations. As rice grain hydrates to more than double its volume whereas navy bean to 


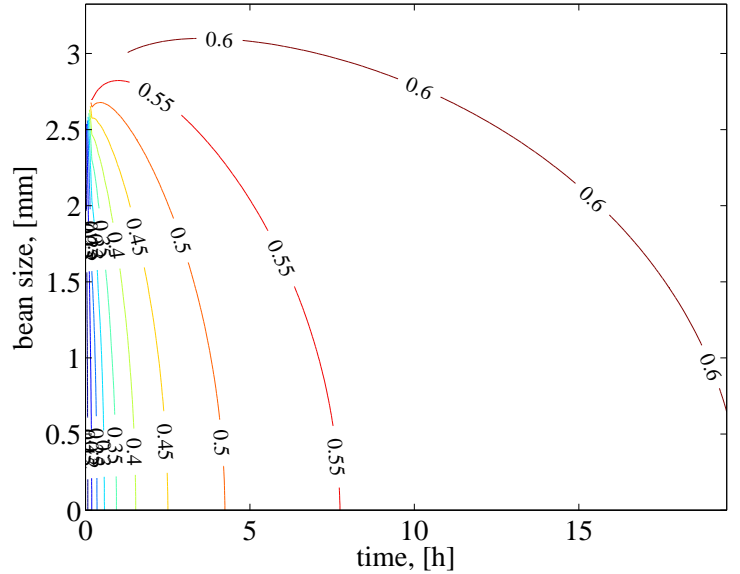

(a) Initial radius $0.0025 \mathrm{~mm}$.

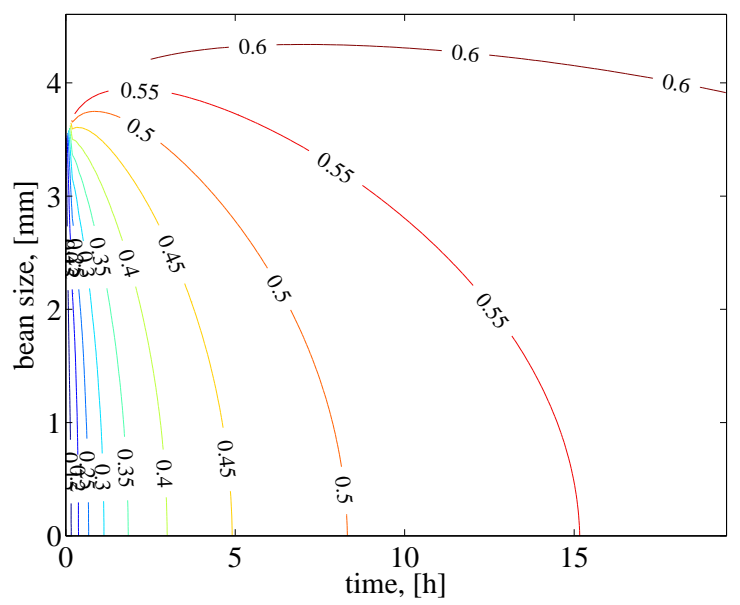

(c) Initial radius $0.0035 \mathrm{~mm}$.

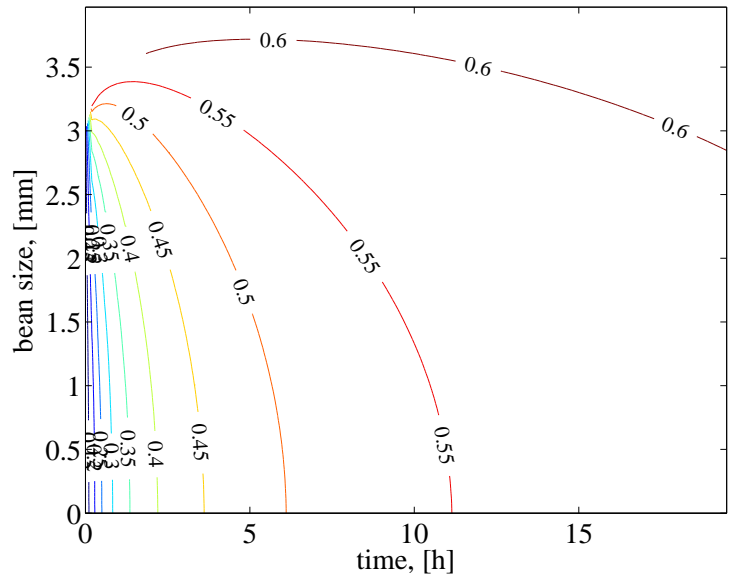

(b) Initial radius $0.003 \mathrm{~mm}$.

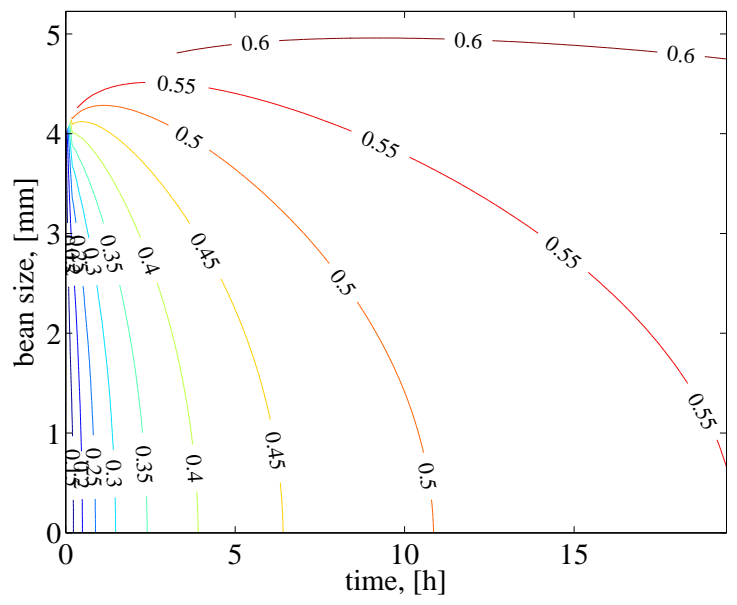

(d) Initial radius $0.004 \mathrm{~mm}$.

Figure 5: Profiles for the bean radius for different initial bean radii taken in the range $[2.5-4.0] \mathrm{mm}$.

approximately 1.2 times the original volume, we take values in the order of $10^{-21} \mathrm{~m}^{2}$. To gauge the sensitivity of the model to permeability value we performed sensitivity analysis in the range $[1.1-5.1] \times 10^{-21} \mathrm{~m}^{2}$. The sensitivity of the moisture content of the bean is presented in Figure 6. It can be observed that the evolution of water saturated pore fraction is directly related to the intrinsic permeability. This indicates the strong coupling between the momentum transport and structure evolution in our model. The model was thus found to be highly sensitive to the selection of permeability values, where a higher permeability indicted a faster rate of hydration. It is worth noting that a non-linearly evolving permeability will allow far more accurate predictions than a constant value. This 
is true considering that the porosity of the bean evolves with time. A direct method for applying the model could be based on the microstructure geometry, similar to that described by Nicolaí and group in their recent work using micro- computed tomography method for fruits (Cantre et al, 2014; Herremans et al, 2014). Conversely, our analysis can also be transformed to an inverse problem scenario to compute the effective water permeability.

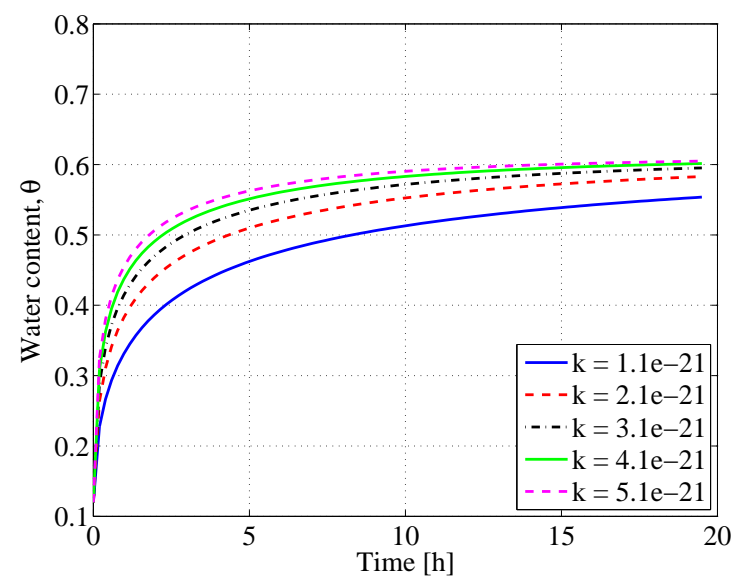

Figure 6: Profiles for the water content for different permeability values taken in the range $[1.1-5.1] \times 10^{-21}$ $\mathrm{m}^{2}$.

\section{Conclusions}

The SCM model demonstrated good predictive capabilities for moisture transport and swelling of the navy beans, without losing the elegance of internal porodynamics. The moving boundary equation accurately predicted the final radii of the bean and supported the rapid creation of free volume inside the bean during rapid hydration phase, followed by structural rearrangements observed in experiments. The model was found sensitive to the selection of the permeability value and modelling on accurate physical domain is highly encouraged. The model presented here is a proof of concept of how hydration of foods can be described by using principles of soft condensed matter physics.

To impart an illustrative value, we simplified the model with some assumptions, so that the theory could be easily discussed. Although we demonstrated the applicability of the model with spherical equivalence and radial profile simulations, the extension of this model 
to foods with complex geometry should be quite straightforward, using finite volume or finite element methods with moving mesh or phase-field and level set approaches using commercial softwares such as COMSOL Multiphysics. This is now practical since significant microstructural details are becoming available with newer imaging techniques such as X-ray micro-computed tomography. The model can be elaborated for cooking of food grains by incorporating the Fourier equation. As a limitation of the current single grain modeling approach, we wish to highlight that diffusion in a single particle is different physically from diffusion through a porous particle layer. Thus, to simulate realistic conditions, the problem turns out to be a two-phase, multiscale, saturated porous media problem. This will be dealt with in our future work.

\section{References}

Abd El-Hady E, Habiba R (2003) Effect of soaking and extrusion conditions on antinutrients and protein digestibility of legume seeds. LWT - Food Science and Technology 36:285-293, DOI 10.1016/s0023-6438(02) 00217-7

Bakalis S, Kyritsi A, Karathanos VT, Yanniotis S (2009) Modeling of rice hydration using finite elements. Journal of Food Engineering 94:321 - 325, DOI 10.1016/j.jfoodeng.2009.03.023

Barry SI, Caunce J (2008) Exact and numerical solutions to a stefan problem with two moving boundaries. Applied Mathematical Modelling 32:83-98, DOI 10.1016/j.apm.2006.11.004

Bellido G, Arntfield S, Scanlon M, Cenkowski S (2003) The effect of micronization operational conditions on the physicochemical properties of navy beans (Phaseolus vulgaris L.). Journal of Food Science 68:17311735, DOI 10.1111/j.1365-2621.2003.tb12320.x

Bello M, Tolaba M, Aguerre R, Suarez C (2010) Modeling water uptake in a cereal grain during soaking. Journal of Food Engineering 97:95-100, DOI 10.1016/j.jfoodeng.2009.09.020

Berg T, Singh J, Hardacre A, Boland MJ (2012) The role of cotyledon cell structure during in vitro digestion of starch in navy beans. Carbohydrate Polymers 87:1678 - 1688, DOI 10.1016/j.carbpol.2011.09.075

Cantre D, East A, Verboven P, Trejo Araya X, Herremans E, Nicolaí BM, Pranamornkith T, Loh M, Mowat A, Heyes J (2014) Microstructural characterisation of commercial kiwifruit cultivars using x-ray micro computed tomography. Postharvest Biology and Technology 92:79-86, DOI 10.1016/j.postharvbio.2014. 01.012

Cozzolino D, Roumeliotis S, Eglinton J (2013) Monitoring water uptake in whole barley (Hordeum vulgare L.) 
grain during steeping using near infrared reflectance spectroscopy. Journal of Food Engineering 114:545549, DOI 10.1016/j.jfoodeng.2012.09.010

Datta A (2006) Hydraulic permeability of food tissues. International Journal of Food Properties 9:767-780, DOI $10.1080 / 10942910600596167$

Davey M, Landman K, McGuinness M, Jin H (2002) Mathematical modeling of rice cooking and dissolution in beer production. AIChE Journal 48(8):1811-1826

Flory PJ (1953) Principles of Polymer Chemistry. Cornell University Press

Ghafoor M, Misra N, Mahadevan K, Tiwari B (2014) Ultrasound assisted hydration of navy beans (Phaseolus vulgaris). Ultrasonics Sonochemistry 21:409-414, DOI 10.1016/j.ultsonch.2013.05.016

Golonka P, Dryzek J, Kluza M (2002) Bean cotyledons microporosity under hydration conditions. Nukleonika 47:137-140

Hamley IW (2007) Polymers, Wiley, chap 2, pp 39-106

Han CC, Akcasu ZA (2011) Dynamics and Kinetics of Phase Separation in Polymer Systems, John Wiley \& Sons, chap 3, pp 103-209. DOI 10.1002/9780470824849.ch3

Herremans E, Verboven P, Defraeye T, Rogge S, Ho QT, Hertog ML, Verlinden BE, Bongaers E, Wevers M, Nicolai BM (2014) X-ray CT for quantitative food microstructure engineering: The apple case. Nuclear Instruments and Methods in Physics Research Section B: Beam Interactions with Materials and Atoms 324:88-94, DOI 10.1016/j.nimb.2013.07.035

Hill TL (1987) Polymer and polyelectrolyte solutions and gels, Dover Publications, chap 21, pp 398-423

Hsu KH (1983) A diffusion model with a concentration-dependent diffusion coefficient for describing water movement in legumes during soaking. Journal of Food Science 48:618-622, DOI 10.1111/j.1365-2621.1983. tb10803.x

Huggins ML (1942a) Some properties of solutions of long-chain compounds. The Journal of Physical Chemistry $46: 151-158$, DOI $10.1021 / \mathrm{j} 150415 \mathrm{a} 018$

Huggins ML (1942b) Theory of solutions of high polymers. Journal of the American Chemical Society 64:1712-1719, DOI 10.1021/ja01259a068

Jin X, van der Sman RGM, Maanen JFC, Deventer HC, Straten G, Boom RM, Boxtel AJB (2014) Moisture sorption isotherms of broccoli interpreted with the flory-huggins free volume theory. Food Biophysics 9:1-9, DOI 10.1007/s11483-013-9311-6

Kereliuk G, Kozub G (1995) Chemical composition of small white (navy) beans. LWT - Food Science and Technology 28:272-278, DOI 10.1016/s0023-6438(95)94176-2

Meyer CJ, Steudle E, Peterson CA (2006) Patterns and kinetics of water uptake by soybean seeds. Journal of Experimental Botany 58:717-732, DOI 10.1093/jxb/erl244

Mezzenga R (2007) Equilibrium and non-equilibrium structures in complex food systems. Food Hydrocolloids 
21:674-682, DOI 10.1016/j.foodhyd.2006.08.019

Mezzenga R, Schurtenberger P, Burbidge A, Michel M (2005) Understanding foods as soft materials. Nature Materials 4:729-740, DOI 10.1038/nmat1496

Mohoric A, Vergeldt F, Gerkema E, de Jager A, van Duynhoven J, van Dalen G, Van As H (2004) Magnetic resonance imaging of single rice kernels during cooking. Journal of Magnetic Resonance 171:157-162, DOI 10.1016/j.jmr.2004.08.013

Mohsenin NN (1986) Physical Properties of Plant and Animal Materials. Routledge

Nicolin D, Coutinho M, Andrade CM, Jorge L (2012) Hsu model analysis considering grain volume variation during soybean hydration. Journal of Food Engineering 111:496-504, DOI 10.1016/j.jfoodeng.2012.02.035

Peleg M (1988) An empirical model for the description of moisture sorption curves. Journal of Food Science 53:1216-1217, DOI 10.1111/j.1365-2621.1988.tb13565.x

Ruiz RS, Vizcarra MG, Martínez C (2008) Hydration of grain kernels and its effect on drying. LWT - Food Science and Technology 41:1310-1316, DOI 10.1016/j.lwt.2007.08.007

van der Sman R (2012) Soft matter approaches to food structuring. Advances in Colloid and Interface Science 176-177:18-30, DOI 10.1016/j.cis.2012.04.002

van der Sman RGM (2007) Soft condensed matter perspective on moisture transport in cooking meat. AIChE Journal 53:2986-2995, DOI 10.1002/aic.11323

van der Sman RGM, van der Goot AJ (2009) The science of food structuring. Soft Matter 5:501, DOI $10.1039 / \mathrm{b} 718952 \mathrm{~b}$

Ubbink J, Mezzenga R (2006) Delivery of functionality in complex food systems: introduction. Trends in Food Science \& Technology 17:194-195, DOI 10.1016/j.tifs.2006.01.004

Ubbink J, Burbidge A, Mezzenga R (2008) Food structure and functionality: a soft matter perspective. Soft Matter 4:1569, DOI 10.1039/b802183j

Wang H, Swain E, Hesseltine C, Heath H (1979) Hydration of whole soybeans affects solid losses and cooking quality. Journal of Food Science 44:1510-1513, DOI 10.1111/j.1365-2621.1979.tb06474.x

Warning A, Verboven P, Nicolaí B, van Dalen G, Datta AK (2014) Computation of mass transport properties of apple and rice from x-ray microtomography images. Innovative Food Science \& Emerging Technologies pp in-press, DOI 10.1016/j.ifset.2013.12.017

Weinstein T, Bennethum L (2006) On the derivation of the transport equation for swelling porous materials with finite deformation. International Journal of Engineering Science 44(18):1408-1422

Winstanley H, Chapwanya M, McGuinness M, Fowler A (2011) A polymer-solvent model of biofilm growth. Proceedings of the Royal Society A: Mathematical, Physical and Engineering Science 467(2129):1449-1467 Zanella-Díaz E, Mújica-Paz H, Soto-Caballero M, Welti-Chanes J, Valdez-Fragoso A (2014) Quick hydration of tepary (phaseolus acutifolius a. gray) and pinto beans (Phaseolus vulgaris L.) driven by pressure 
